\title{
Advanced Spectrum Management for the Downlink of WCDMA Systems using Genetic Algorithms
}

\author{
J. Nasreddine, J. Pérez-Romero, O. Sallent \\ TSC department, Universitat Politècnica de Catalunya (UPC) \\ c/ Jordi Girona, 1-3, Campus Nord, Barcelona, Spain \\ e-mail: [jnassred, jorperez, sallent]@tsc.upc.edu)
}

\begin{abstract}
In this paper, we propose an advanced spectrum management approach for the downlink of WCDMA systems using genetic algorithms and the concept of coupling matrix, which is able to capture inter-cell interactions. Simulation results show that the proposed approach increases spectrum efficiency while guaranteeing the required QoS levels. Moreover, it allows releasing carriers in some cells, so that they could be eventually used by e.g. secondary cognitive radio users exploiting the flexible spectrum allocation and opportunistic spectrum access.
\end{abstract}

Index Terms- coupling matrix; advanced spectrum management; genetic algorithms; WCDMA

\section{INTRODUCTION}

Current radio spectrum allocation is based on static allocation of the spectrum to specific purposes. This allocation leads to an inefficient and unbalanced use of the spectrum since some networks suffer from spectrum scarcity while the assigned spectrum to other networks is significantly underutilized. In that sense, the Federal Communications Commission (FCC) stated that spectrum access and not spectrum scarcity reduces spectrum efficiency [1]. The inefficiency in spectrum usage, together with the growing competition for spectrum, requires a new spectrum allocation paradigm that breaks the spectrum access limitation and enables opportunistic access to the spectrum. Therefore, the regulatory perspective on how the spectrum should be allocated and utilized in future wireless scenarios is evolving towards a cautious introduction of more flexibility in the spectrum management together with economic considerations on spectrum trading [2][3]. Then, instead of the classical fixed spectrum allocation to licensed systems and services for a long period, the possibility to use flexible radio spectrum management strategies that dynamically allocate spectrum bands in accordance with the specific traffic needs in each area is being recently considered [4]-[6]. In this framework, several steps can be envisaged in the path towards a completely flexible use of the spectrum. In particular, one of the initial possibilities relies on the coexistence of primary and secondary networks [7][8]. A

This work was performed in project $E^{3}$ which has received research funding from the Community's Seventh Framework program. This paper reflects only the authors' views and the Community is not liable for any use that may be made of the information contained therein. The contributions of colleagues from $E^{3}$ consortium are hereby acknowledged. This work has been also supported by the Spanish Research Council and FEDER funds under COGNOS grant (ref. TEC2007-60985). primary network owns a license to use a spectrum block, whereas a secondary network can opportunistically use this block with hard constraints on transmitted power. Herein, two key questions to answer are, 'How the primary network can release some of its carriers?' and 'How the secondary network can find, in the fastest way, the best block of spectrum that can satisfy its QoS constraints while respecting primary constraints?'. The answer to the first question depends on the type of used access technologies and interference characteristics whereas the answer to the second question depends on different aspects such as the level of collaboration between primary and secondary networks, the type of primary and secondary applications, secondary network capabilities, etc. In this paper, we focus on the first question considering the downlink of WCDMA system as a primary network and propose a genetic algorithm-based Advanced Spectrum Management (ASM) approach using the coupling matrix properties presented in [9].

In the last few years, several ASM approaches have been proposed. They mainly differ by the used allocation algorithms or the used metrics to reflect system performance. Some approaches use traffic estimators to predict the needed number of carriers without a proper representation of interference [11][12]. Other approaches consider that the relation between inter-cell and intra-cell interferences is independent from the spectrum allocation [13], which is not true in interference limited systems. Hence, inter-cell interference can be wrongly estimated leading to non-suitable allocations. A more accurate inter-cell interference representation has been proposed in [9] using coupling matrix properties. In this context, this paper introduces a new ASM approach for the downlink of WCDMA systems which has different characteristics than the uplink and therefore requires different approach than the one presented in [14]. Moreover, Genetic Algorithms (GA) are chosen for the allocation algorithm due to their fast convergence and the possibility of obtaining multiple solutions [10].

The remaining of this paper is organized as follows. First, we introduce the coupling matrix in section II. The optimization problem related to the ASM is defined in section III and the genetic algorithm-based ASM approach is elaborated in section IV. Then, the performance of the proposed algorithm is studied in section V. Finally, we 
conclude with useful remarks and future works.

\section{COUPLING MATRIX}

We consider a WCDMA system with $K$ cells and $F_{\text {all }}$ carriers. The set of allocated carriers to the system is called $\Phi=\left\{f: f \in\left\{1,2, \ldots, F_{\text {all }}\right\}\right\}$ and the set of all cells is called $\Lambda=\{j: j \in\{1,2, \ldots, K\}\}$. We call $\Lambda^{(f)}$ the set of cells using carrier $f, F_{j}$ the number of carriers allocated to cell $j$ and $A=\left\{\Lambda^{(1)}, \Lambda^{(2)}, \cdots, \Lambda^{\left(F_{\text {all }}\right)}\right\}$ the carrier allocation to cells.

Coupling matrix has been introduced in [9] and it is computed by assessing a relationship between the total received powers in downlink using $E_{\mathrm{b}} / N_{\mathrm{o}}$ definition:

$$
\left(E_{b} / N_{o}\right)_{i_{j}}=\frac{\Theta_{i_{j}} \times P_{T, i_{j}} / L_{i_{j}, j}}{N_{T}+\chi_{i_{j}}+\alpha \times\left(P_{T, j}-P_{T, i_{j}}\right) / L_{i_{j}, j}}
$$

where $P_{T, i_{j}}$ is the power devoted to mobile $i_{j}$ of cell $j$, $\chi_{i_{j}}$ represents the inter-cell interference experienced by $i_{j}$, $\Theta_{i_{j}}$ is the spreading factor of $i_{j}$ (i.e. the ratio between chip rate and bit rate), $L_{i_{j}, j}$ is the path loss between mobile $i_{j}$ and base station $j$ including antenna gains, $\alpha$ is the orthogonality factor ( $\alpha=0$ for perfect orthogonality and 1 for non-orthogonality), $N_{T}$ is the background noise power and $P_{T, j}$ is the total transmitted power by base station $j$ that is given by

$$
P_{T, j}=P_{p, j}+\sum_{i_{j}=1}^{n_{j}} P_{T, i_{j}}
$$

where $n_{j}$ is the number of users connected to cell $j$ and $P_{p, j}$ is the power devoted to common control channels in cell $j$.

It was shown in [9] that, for a given carrier, the vector of total transmitted powers $\boldsymbol{P}=\left(P_{1}, P_{2}, \ldots, P_{K}\right)^{\mathrm{T}}$ satisfying $E_{\mathrm{b}} / N_{\mathrm{o}}$ constraints can be written in the following matrix form:

$$
\boldsymbol{P}=\boldsymbol{C P}+\boldsymbol{P}_{N}
$$

where $\boldsymbol{C}$ and $\boldsymbol{P}_{\boldsymbol{N}}$ are respectively the $K \mathrm{x} K$ coupling matrix and the $K \times 1$ noise vector defined by the elements:

$$
C_{j, l}=\left\{\begin{array}{ll}
0 & \text { if } l=j \\
\frac{S_{j, l}}{1-\alpha S_{j, j}} & \text { otherwise }
\end{array} \text { and } P_{N, j}=\frac{P_{p, j}+N_{T} Z_{j}}{1-\alpha S_{j, j}}\right.
$$

where $S_{j, l}$ and $Z_{j}$ are defined by:

$$
S_{j, l}=\sum_{i_{j}=1}^{n_{j}} \frac{1}{\varepsilon_{i_{j}}} \frac{L_{i_{j}, j}}{L_{i_{j}, l}} \quad \text { and } \quad Z_{j}=\sum_{i_{j}=1}^{n_{j}} \frac{1}{\varepsilon_{i_{j}}} L_{i_{j}, j} .
$$

where $\varepsilon_{i_{j}}$ is defined by: $\varepsilon_{i_{j}}=\alpha+\Theta_{i_{j}} /\left(E_{\mathrm{b}} / N_{\mathrm{o}}\right)_{i_{j}}$.

Each element of matrix $\boldsymbol{C}$ represents the variation of the total transmitted power by one cell as a response to the variation in the total transmitted power by another cell.

In a system with several carriers, a different coupling matrix is associated to each carrier. The associated matrix to carrier $f$ is called $\boldsymbol{C}^{(f)}$ and only includes the elements corresponding to cells of set $\Lambda^{(f)}$ that are associated to carrier $f$. Therefore, the different carriers will generally have coupling matrices with different sizes. In order to map the cell indices of the total coupling matrix $\boldsymbol{C}$ to the indices of matrix elements in the different carriers, we define the set of mapping relations $\mathcal{M}_{f}$ :

$$
\mathcal{M}_{f}(j)=j-K_{f, j}
$$

where $K_{f, j}$ is the number of cells that are not associated to carrier $f$ and have a smaller index than $j$ :

$$
K_{f, j}=\left|\left\{l \in \Lambda-\Lambda_{f}: l<j\right\}\right|
$$

where $|$.$| is the cardinality of a set. Thus, the corresponding$ element reflecting the interaction between cell $j$ and $l$ in $C^{(f)}$ is $C_{\mathcal{M}_{f}(j), \mathcal{M}_{f}(l)}^{(f)}$ and is computed in a system where users of a cell are considered to be uniformly distributed over carriers reflecting a load balancing strategy (i.e. the same load is kept in all carriers). Therefore, the value of the factors $S_{\mathcal{M}_{f}(j), \mathcal{M}_{f}(l)}^{(l)}$ can be written as:

$$
S_{\mathcal{M}_{f}(j), \mathcal{M}_{f}(l)}^{(f)}=S_{j, l} / F_{j} .
$$

Then, the coupling matrix and the noise power vector are estimated using (4) and (8):

$$
C_{\mathcal{M}_{f}(j), \mathcal{M}_{f}(l)}^{(f)}=\frac{S_{j, l} / F_{j}}{1-\alpha S_{j, j} / F_{j}} \quad \text { and } \quad P_{N, \mathcal{M}_{f}(j)}^{(f)}=\frac{P_{p, j}+N_{T} Z_{j} / F_{j}}{1-\alpha S_{j, j} / F_{j}}
$$

\section{PROBLEM FORMULATION}

A network operator will intend to get the maximum revenue out of its deployed infrastructure and the gained spectrum usage rights by maximizing the utilization of the available radio resources, which are the result of the deployed sites and allocated carriers per cell in a given area. Ideally, the amount of radio resources at a given time and place should match traffic demand. However, this is not possible in practice with a fixed site deployment and carrier allocation, since the high level of dynamism associated to traffic demand leads to mismatches between offered and requested radio resources. Then, focusing on time scales ranging from minutes to hours and assuming that, on the one hand, the site deployment is fixed and, on the other hand, proper short-term radio resource management strategies are operating, ASM will be the technique enabling the necessary flexibility to achieve the best possible matching between offered and required spectrum resources by adding or removing carriers to/from cells in accordance with user density, environment characteristics, service type and connection establishment processes. In turn, the observation that some user satisfaction and/or network performance metrics are degraded compared to some reference values can be taken as a trigger event for the case that the current amount of carriers should be increased. On the contrary, the observation that these metrics are enhanced compared to some reference values can be taken as a trigger event for the release of some carriers.

In this context, our objective is to minimize the number of allocated carriers to cells while conserving an acceptable QoS level. Therefore, we use the following definition of spectrum 


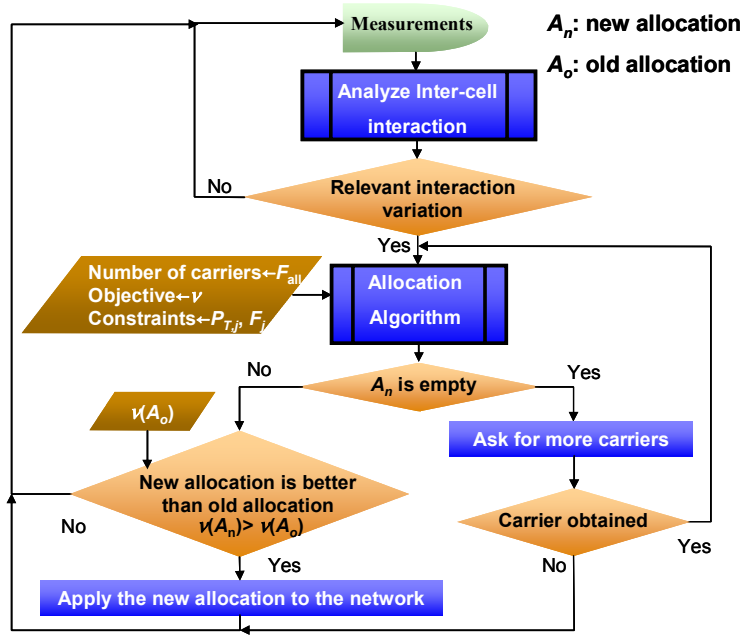

Fig. 1. High level vision of the ASM approach

efficiency when only one service is provided by the operator and that represents cell throughput per unit of bandwidth:

$$
v=R_{b} \frac{1}{K W} \sum_{j \in \Lambda} \frac{n_{j}}{F_{j}} .
$$

where $W$ is the WCDMA bandwidth and $R_{b}$ is the bit rate.

When a triggering event is detected, the allocation algorithm is performed to find the best carrier allocation to cells that find the best solution to the following optimization problem:

$$
\begin{array}{lll}
\text { Maximize } & v & \\
\text { Subject to } & P_{T, j} \leq \beta P_{\max , j} & \forall j \in \Lambda \\
& F_{j} \leq F_{\text {all }} & \forall j \in \Lambda
\end{array}
$$

where $\beta$ is the objective ratio of the total available transmitted power at cell $j$ to take into account a margin for fading effect $(0<\beta<1)$ and $P_{\max , j}$ is the maximum available power at cell $j$.

\section{PROPOSED APPROACH}

A high level diagram of the proposed approach is presented in Fig. 1. First, the information from radio measurements is collected to detect substantial variations in the scenario in terms of spatial traffic distribution and load level, which are reflected by modifications in inter-cell interactions. For that purpose, metrics as the coupling matrix that are able to capture such interactions are required. By analyzing coupling matrix properties, it is possible to detect the relevant variations and trigger the allocation algorithm to determine if more carriers are required in some cells (e.g. if the traffic load has increased and it can no longer be served with the current number of carriers) or if some carriers can be released (e.g. if the traffic load has decreased and it can be served with fewer carriers).

Thereafter, the allocation algorithm choose a carrier allocation $A_{n}$ in accordance with the optimization problem defined in (11). If no carrier allocation satisfies the constraints, the optimization problem does not have a feasible solution and the new allocation $A_{n}$ at the output of the algorithm will be empty. This means that, with the actual number of carriers, the system will not be able to handle the traffic with the specified
QoS requirements. In this case, the ASM can try to obtain more carriers, e.g. by requesting a carrier to the regulator or another operator that has released some carriers for a secondary usage. If this step fails, the ASM keeps the old allocation $A_{o}$. In turn, if a new carrier is obtained, the allocation algorithm will be executed again.

In case that the algorithm provides a feasible allocation (i.e. the new allocation $A_{n}$ at the output of the algorithm is not empty), meaning that QoS requirements can be fulfilled, the ASM tests if the new allocation has better spectrum efficiency than the actual allocation (i.e. if $v\left(A_{n}\right)>v\left(A_{o}\right)$ ). If so, the new allocation is applied to the network and the unused carriers can be put in secondary market. Otherwise, the ASM keeps the previous carrier allocation $A_{o}$ to reduce signaling traffic.

\section{A. Detecting Relevant Interaction Variations}

As for the inter-cell interaction detection phase in Fig. 1, it is based on coupling matrix $\boldsymbol{C}$ and noise power $\boldsymbol{P}_{N}$, which are computed using (4) and (5) based on measurements and/or outputs from planning tools. A relevant interaction variation is detected based on two conditions: (1) the measured percentage of users not reaching the $E_{b} / N_{\mathrm{o}}$ requirements is higher than a given threshold in at least one cell, (2) the difference between $r$ elements of the aggregated coupling matrices in two observation periods is higher than a given threshold.

\section{B. Genetic Allocation Algorithm}

The optimization problem (11) is an NP-hard problem; therefore, we propose a binary GA to solve the problem. GAs are search techniques used to find near-optimum solutions for hard optimization problems. GAs have been used in allocation algorithms but with different metrics than coupling matrix as in [13]. These algorithms are based on the principles of genetics and natural selection, where a starting population will lead to a stronger population after series of procedures such as selection, crossover and mutation. Each allocation is represented in the binary GA by a binary string of 0 and 1 in the encoding process. The convergence speed and the obtained results depend on the used encoding scheme.

The allocation algorithm starts by a first estimation of the minimum number of carriers to be allocated to each cell. In cell $j$, the needed number of carriers should be sufficient to handle at least the load coming from intra-cell users, which corresponds to parameter $S_{\mathcal{M}_{f}(j), \mathcal{M}_{f}(j)}^{(f)}$. This means that the following condition should be fulfilled [15]:

$$
S_{\mathcal{M}_{f}(j), \mathcal{M}_{f}(j)}^{(f)} \leq 1 \quad \forall f \in \Phi_{j}
$$

where $\Phi_{j}$ is the set of carriers allocated to cell $j$ : $\Phi_{j}=\left\{f: j \in \Lambda^{(f)}\right\}$.

The estimation of the minimum number of carriers $F_{j, \text { min }}$ for cell $j$ is first initiated by combining (8) and (12):

$$
F_{j, \min }=\left\lceil S_{j, j}\right\rceil \text {. }
$$

where $\left\lceil S_{j, j}\right\rceil$ is the first integer higher or equal than $S_{j, j}$. 
Then, we test if the system can handle the intra-cell interference using $F_{\text {all }}$ (i.e. $F_{j, \text { min }}$ is lower or equal to $F_{\text {all }}$ ). If this test fails, the algorithm will stop and generate output $A_{n}$ equal to an empty set. This means that the network requires more carriers for a proper operation. Otherwise, the algorithm enters in the GA loop where the solution space involves all allocations that verify the following two constraints:

$$
\begin{array}{ll}
F_{j} \leq F_{\text {all }} & \forall j \in \Lambda \\
F_{j} \geq F_{j, \text { min }} & \forall j \in \Lambda
\end{array} .
$$

It is worth here to define the different parameters of the GA that starts by generating a population of chromosomes. In this paper, we use an $F \times K$ binary matrix $c$ to represent an allocation $A$, where each line corresponds to a carrier and each column to a cell. Hence, element $c_{f, j}$ is set to 1 if carrier $f$ is allocated to cell $j$ and to 0 otherwise. This encoding is necessary in order to obtain the generated chromosomes by a crossover in the solution space as it will be shown afterward.

In order to decrease convergence time, we use an initial population including 12 chromosomes corresponding to:

1) Two allocations where the first carrier will be allocated to all cells, the second to all cells using at least two carriers, the third to all cells using at least three carriers, and so on. In the allocation, each cell uses only the minimum number of carriers given by (13). In the second allocation, one carrier is added for each cell if possible to give a margin for inter-cell interference.

2) An allocation where each carrier is allocated to all cells.

3) Eight random allocations satisfying (14).

The chromosomes are evaluated and ranked according to a fitness function that will be introduced in subsection $C$. In the fitness function, fitness factor $\phi_{c}$ is associated to chromosome $c$ that represents the suitability of the chromosome.

In order to generate a better population, only the $M$ best chromosomes are conserved and the 12- $M$ remaining chromosomes are discarded. From the $M$ chromosomes, 12- $M$ parents are selected to breed new children with ameliorated fitness factors, where chromosome $c$ is chosen based on a roulette wheel selection with probability $p_{s, c}$ :

$$
p_{s, c}=\frac{\phi_{c}}{\sum_{c=1}^{10} \phi_{c}} \text {. }
$$

In the proposed algorithm, we consider that $M$ is equal to 8 . The four children chromosomes are generated by a single point crossover process over six parent chromosomes as shown in Fig. 2. In the generated chromosomes, the number of allocated carriers to each cell is conserved whereas the associated cells to a given carrier may change. Since the parents are in the space of solution, this type of crossover allows the generation of chromosomes in the space solution to increase convergence speed. Moreover, the seventh and the eight ranked chromosomes are replaced by the following chromosomes:

1) The seventh chromosome is obtained from the best

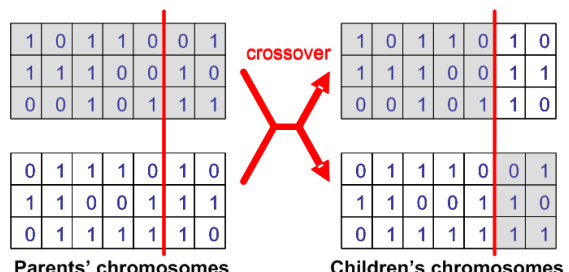

Fig. 2. Crossover between two chromosomes in a system with 7 cells and 3 carriers

allocation (i.e. with the highest $\phi_{c}$ ) by reducing the number of carriers allocated to a random set of cells without breaking the constraint (14). This allows, when possible, to obtain higher spectrum efficiency.

2) The eighth chromosome is generated randomly in the solution space in order to increase system entropy.

In order to change the number of carriers allocated to a cell, the mutation process is applied to all chromosomes except the first and the seventh chromosomes that have the best fitness factors. The mutation involves the change of a bit in a chromosome with a probability $p_{m}$ in order to maintain genetic diversity from one generation to the next one.

\section{Fitness Function}

The presented processes for the GA, except the fitness function, are generic and can be applied using any performance metric. In this paper, we propose two fitness functions that will be compared to a reference fitness function. The two fitness functions are called respectively: Coupling Matrix Properties-driven Fitness Function (CMP-FF) and Coupling Matrix Elements-driven Fitness Function (CMEFF). The three functions differ by the way total transmitted power vector in a given carrier is computed. After the computation of the latter, the fitness factor is obtained by:

$$
\phi_{c}=5 \arctan (0.1 u)+\pi / 2
$$

where $u$ is defined by the following equation:

$$
u= \begin{cases}\sum_{f=1}^{F_{\text {all }}} \sum_{j \in \Lambda^{(f)}} n_{j}^{(f)} \min \left(0, P_{T, j}^{(f)}-\beta P_{\max , j}\right) & \text { if } \exists P_{T, j}^{(f)}>\beta P_{\max , j} \\ v & \text { otherwise }\end{cases}
$$

This fitness factor has a range between 0 and $3 \pi$, and it is higher than $\pi / 2$ when transmitted power constraint is satisfied. Moreover, it is an increasing function of $v$ and a decreasing function of the exceeded transmitted power. This allows penalizing more the chromosomes with high transmitted powers. Finally, a correction factor is added to the fitness factor in case it is the same for different chromosomes due to the fact that different allocations may have the same spectrum efficiency while one of these allocations has a fully released carrier. The corrected fitness factor is given by:

$$
f_{a, c}=f_{c}+\frac{R_{c}}{\sum_{c=1}^{10} R_{c}}\left(\phi_{c}-\phi_{u_{c}}\right)
$$

where $R_{c}$ is the number of fully released carriers in chromosome $c$ and $u_{c}$ is the chromosome whose fitness function is the first fitness function that is higher than the 
fitness of chromosome $c$. The factor $\left(\phi_{c}-\phi_{u_{c}}\right)$ is added so that the addition of the corrective factor does not change the ranking of the chromosomes with different fitness factors.

\section{Reference Fitness Function}

In the reference fitness function which is similar to the one proposed in [13], the transmitted power by base station $j$ using carrier $f$ is computed as follows [15]:

$$
P_{T, j}^{(f)}=\frac{P_{p, j}+N_{T} Z_{j}}{1-\eta_{j}^{(f)}}
$$

where $\eta_{j}^{(f)}$ is the load factor at carrier $f$. By using the same approximations as in [16], we get:

$$
P_{T, j}^{(f)}=\frac{N_{T} \overline{L_{j}^{(f)}}}{1-\eta_{j}^{(f)}} \sum_{i_{j} \in \xi_{j}^{(f)}} \frac{\left(E_{\mathrm{b}} / N_{\mathrm{o}}\right)_{i_{j}}}{\Theta_{i_{j}}}+\frac{P_{p, j}}{1-\eta_{j}^{(f)}}
$$

where $\xi_{j}^{(f)}$ is the set of mobiles using carrier $f$ in cell $j, \overline{L_{j}^{(f)}}$ is the average path loss between cell $j$ and its mobiles using carrier $f$, and

$$
\eta_{j}^{(f)}=\sum_{i_{j} \in \xi_{j}^{(f)}} \frac{\left(E_{\mathrm{b}} / N_{\mathrm{o}}\right)_{i_{j}}}{\Theta_{i_{j}}}\left(\beta_{j}+\kappa_{j}\right)
$$

where $\kappa_{j}$ is the inter-cell to intra-cell ratio.

\section{Coupling Matrix Properties-driven Fitness Function}

In the CMP-FF, $\boldsymbol{P}^{(f)}$ is computed using (3) [9]:

$$
\boldsymbol{P}^{(f)}=\left(\mathfrak{I}_{\left|\Lambda^{(f)}\right|}-\boldsymbol{C}^{(f)}\right)^{-1} \boldsymbol{P}_{N}^{(f)}
$$

where $\mathfrak{I}_{\left|\Lambda^{(f)}\right|}$ is the $\left|\Lambda^{(f)}\right| \times\left|\Lambda^{(f)}\right|$ identity matrix.

The computed power is positive only when the spectral radius of matrix $\boldsymbol{C}^{(f)}$ is lower than 1 [9]. Therefore, the spectral radius is tested before applying (22). If it is higher than 1, all transmitted powers are set to the maximum available power.

\section{Coupling Matrix Elements-driven Fitness Function}

The CME-FF is a relaxed function of the CMP-FF where the transmitted power by a base station $j$ for carrier $f$ is computed using (3) and considering that all cells transmits the same portion of their maximum powers:

$$
P_{T, j}^{(f)}=\frac{N_{T} P_{\max , j}}{P_{\max , j}-\sum_{l \in \Lambda^{(f)}} C_{j, l} P_{\max , l}}
$$

The condition on spectral radius is transformed here to the condition that the denominator of (23) is positive.

\section{Simulations AND Results}

The GA with the three fitness functions is evaluated in a macro-cell system with four rings in addition to five hotspots randomly distributed inside the central cell. Mobile density in hotspots is 15 times higher than in macro-cells. Simulation parameters are introduced in Table 1. We assume that the operator has 3 carriers at the beginning of simulations. All
TABLE 1.

SIMULATION PARAMETERS

\begin{tabular}{ll}
\hline \hline Macro-cell radius & $1 \mathrm{~km}$ \\
hotspot radius & $200 \mathrm{~m}$ \\
Path loss model & $128.1+37.6 \times \log _{10} d(\mathrm{Km})$ \\
Background noise power & $-98 \mathrm{dBm}$ \\
Maximum allowed power in macro & $43 \mathrm{dBm}$ \\
Maximum allowed power in micro & $33 \mathrm{dBm}$ \\
Transmitted power range & $25 \mathrm{~dB}$ \\
Pilot channel power & $15 \%$ of $P_{j, \text { max }}$ \\
$E_{\mathrm{b}} / N_{\mathrm{o}}$ target & $4.6 \mathrm{~dB}$ \\
Spreading factor $\Theta$ & $25 \mathrm{~dB}$ \\
Orthogonality factor $\alpha$ for marco & 0.4 \\
Orthogonality factor $\alpha$ for mirco & 0.06 \\
Shadowing factor deviation & $7 \mathrm{~dB}$ \\
Shadowing factor cross-correlation & 0.5 \\
Power control & Perfect power control \\
$\beta$ & 0.8
\end{tabular}

needed information $\left(E_{b} / N_{\mathrm{o}}\right.$, long-term path losses, etc.) for the computation of the coupling matrices can be obtained using the measurements collected either by cells or mobiles in an operative network. Herein, we assume that the measurement phase has been already done and we only evaluate the performance of the allocation algorithm. It should be noted that detection mechanisms were evaluated in [9] and [17].

In Fig. 3 and Fig. 4, the spectrum efficiency and the normalized maximum power over cells are plotted as a function of mobile density for the GA algorithm using the two proposed fitness functions and the reference function. The total transmitted power is normalized using the objective allowed transmitted power $\beta P_{\max , j}$. It can be seen from these results that the proposed fitness function can increase the spectrum efficiency by up to $45 \%$ compared to the reference function when $\kappa$ is different than 0.4 . In that last case, the reference function gives approximately the same efficiency than the CME-FF. However, the transmitted power cannot be controlled efficiently and can be more than the acceptable threshold in some cases as shown in Fig.4. Therefore, this case is discarded because it cannot guarantee the QoS. The bad performance of the reference method is due to the fact that for low values of $\kappa$, the inter-cell interference can be underestimated together with the number of needed carriers. Moreover, high values of $\kappa$ can give an overestimation of inter-cell interference and leads to a decrease in spectrum efficiency. Therefore, the value of $\kappa$ should be optimized for each spectrum allocation in order to give good results. Moreover, the GA algorithm gives slightly better spectrum efficiency when the CMP-FF is used compared to the case when the CME-FF is used. These performance ameliorations are due to the precise estimation of the total transmitted power. Moreover, we can see that the proposed functions always satisfy power constraints. The transmitted power, when CMP-FF is used, is near to the maximum allowed but without reaching it. This leads to a maximum exploitation of the allocated carriers. Finally, the number of iterations, when the CMP-FF is used, changes slightly if user density increases and it is around 30 iterations, whereas the number of iterations when CME-FF or the reference functions has high variance and changes between 200 and 300 with CMP-FF and between 


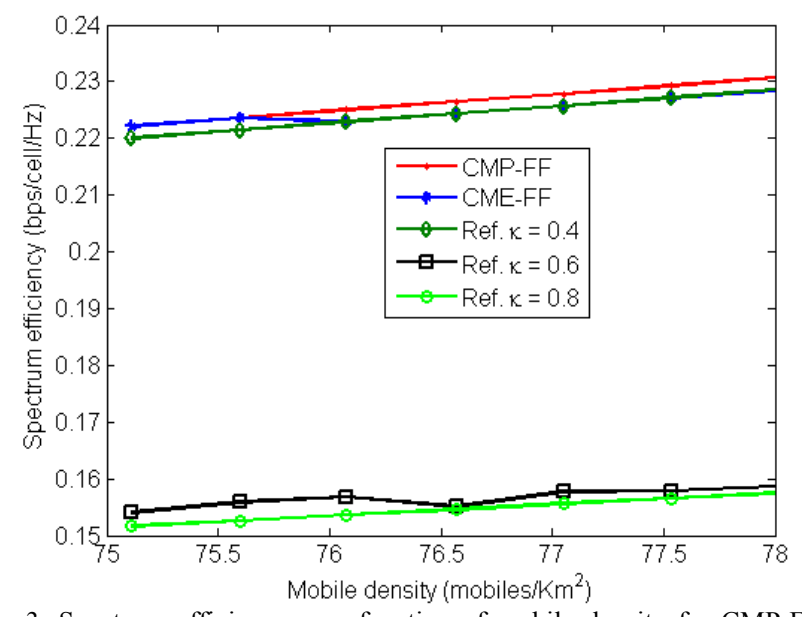

Fig. 3. Spectrum efficiency as a function of mobile density for CMP-FF, CME-FF and reference method with $\kappa=0.4,0.6$ and 0.8

50 and 800 when the reference function is used. It should be noted that the complexity of one CMP-FF iteration is $O\left(K^{3}\right)$ if the power method is used, whereas the complexity in the other cases is $O(K)$. Therefore, the convergence of the GA algorithm when CMP-FF is used can be significantly slower than when the reference function is used in big systems, while the CMEFF gives similar results with lower complexity. Therefore, a tradeoff can be taken by the operator between complexity and spectrum efficiency. It should be noted here that the ASM approach is applied at a rather medium to long term scale; therefore the complexity of the three solutions can be supported.

\section{CONCLUSIONS AND FUTURE WORK}

In this paper, we have introduced a centralized ASM algorithm for WCDMA networks using genetic algorithms. The proposed approach is based on the utilization of coupling matrix properties to reduce inter-cell interactions. In this context, we defined two new fitness functions based on different ways of the estimation of the transmitted powers. Simulation results have shown that these functions lead to interesting results in terms of guaranteeing QoS levels reflected by the maximum transmitted power and increasing spectrum efficiency. The increase in spectrum efficiency for a fixed number of users reflects an increase in the number of released carriers. This leads to a higher possibility that a secondary network could use the released carriers. Moreover, we have noticed that the relaxed function give similar results to the precise function with lower complexity. Therefore, the former can be used in case of highly complex systems.

Our future work focuses on the utilization of the released carriers by cognitive radios without polluting WCDMA users with harmful interference.

\section{REFERENCES}

[1] FCC, "Report of the Spectrum Efficiency Working Group," Spectrum Policy Task Force, Nov. 2002.

[2] J. A. Hoffmeyer, "Regulatory and Standardization Aspects of DSA Technologies - Global Requirements and Perspectives", IEEE DySPAN, Baltimore, MD, November, 2005.

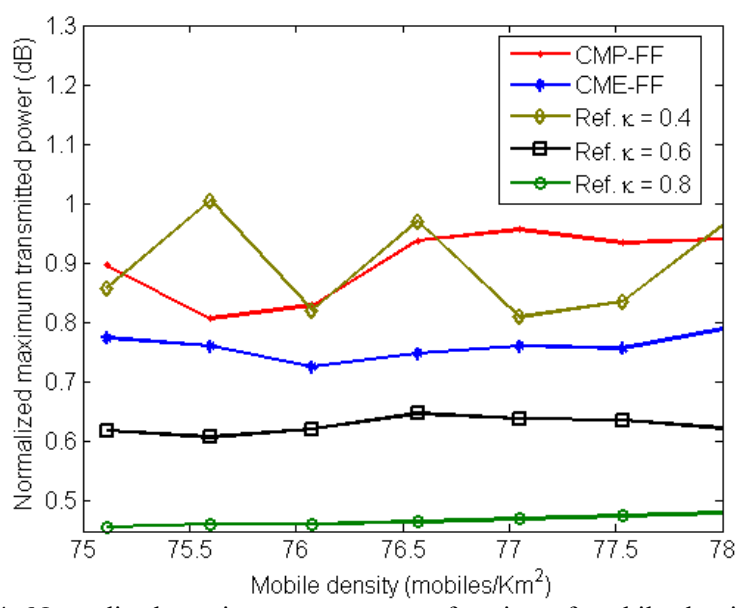

Fig. 4. Normalized maximum power as a function of mobile density for CMP-FF, CME-FF and reference method with $\kappa=0.4,0.6$ and 0.8

[3] Spectrum Usage Rights, Technology and usage neutral access to the radio spectrum, Ofcom Consultation document, 12 April 2006.

[4] M. Buddhikot, P. Kolodzy, S. Miller, K. Ryan, J. Evans “ DIMSUMNet: New Directions in Wireless Networking Using Coordinated Dynamic Spectrum Access", IEEE WoWMoM, June, 2005.

[5] M. Buddhikot, K. Ryan, "Spectrum Management in Coordinated Dynamic Spectrum Access Based Cellular Networks", IEEE DySPAN, Baltimore, MD, November, 2005.

[6] H. R. Karimi, T.W.Ho, H. Claussen, L. G. Samuel, "Evolution towards dynamic spectrum sharing in mobile communications", IEEE PIMRC, Helsinki, September, 2006.

[7] S. Panichpapiboon, J.M. Peha "Providing secondary access to licensed spectrum", Wireless Networks journal, Springer, October, 2006.

[8] S. M Mishra, R. Tandra, A. Sahai, "Coexistence with Primary Users of Different Scales," IEEE DySPAN, 2007, 158-167

[9] J. Nasreddine, O. Sallent, J. Pérez-Romero, R. Agusti, "Novel Inter-Cell Interaction Approach for WCDMA-based Cognitive Networks", IEEE ICC, Glasgow-Scotland, June 2007.

[10] R. L. Haupt, S. E. Haupt, Practical Genetic Algorithms, Willey, 2004.

[11] V. Rodriguez, K. Moessner, R. Tafazolli1, "Market Driven Dynamic Spectrum Allocation over Space and Time among Radio-Access Networks: DVB-T and B3G CDMA with Heterogeneous Terminals," Mobile Networks and Applications, vol .11, n. 6, 2006, p.p 847-860.

[12] MK Pereirasamy, J Luo, M Dillinger, C Hartmann, "An approach for interoperator spectrum sharing for 3G systems and beyond," IEEE PIMRC, vol.3, pp. 1952-1956, 2004.

[13] D. Thilakawardana, K. Moessner, "Enhancing Spectrum Productivity through Cognitive Radios facilitating Cell-by-Cell Dynamic Spectrum Allocation", Software Defined Radio Technical Conference, 2007.

[14] J. Nasreddine, J. Pérez-Romero, O. Sallent, R. Agusti, "Simulated Annealing-Based Advanced Spectrum Management Methodology for WCDMA Systems," IEEE ICC, 2008.

[15] J. Pérez-Romero, O. Sallent, R. Agusti and M. A. Diaz-Guerra, Radio Resource Management strategies in UMTS, John wiley \& Sons, 2005.

[16] H. Holma, A. Toskala, WCDMA for UMTS: HSPA Evolution and LTE, $4^{\text {th }}$ edition, John wiley \& Sons, 2007.

[17] J. Nasreddine, J. Pérez-Romero, O. Sallent, R. Agusti ,"Dynamic Spectrum Management Methodology for WCDMA Systems Based on Inter-Cell Interaction Approach", WPMC, September 2006 